\title{
Bleeding in a patient taking Lorenzo's oil: evidence for a vascular defect
}

\author{
BC Chai, WS Etches, MW Stewart, K Siminoski
}

\begin{abstract}
Summary
We describe a man with adrenoleukodystrophy receiving Lorenzo's oil (glycerol trioleate and glycerol trierucate) who developed purpura, petechiae, and bleeding. Bleeding time was markedly increased $(>20 \mathrm{~min})$, although he had only borderline thrombocytopenia $\left(120 \times 10^{9} / 1\right)$ and conventional platelet aggregation studies were normal (except for a borderline response to low concentration collagen), as were results using a new technique employing immobilised von Willebrand factor. Together these results suggest that bleeding in this man resulted from a defect in vascular wall function or in the interaction of platelets with the endothelium.
\end{abstract}

Keywords: adrenoleukodystrophy, platelets, fatty acids, bleeding time

In adrenoleukodystrophy there is tissue accumulation of very-long-chain fatty acids (VLCFA), probably resulting from a deficiency of lignoceroyl-CoA ligase activity. An experimental dietary therapy restricts VLCFA intake and provides supplementation with long-chain fatty acids (LCFA) in the form of Lorenzo's oil (a combination of glycerol trioleate and glycerol trierucate). ${ }^{1,2}$ Recently this regimen has been found to induce thrombocytopenia, although significant clinical bleeding has not been reported as a consequence. ${ }^{3,4}$ We describe a man with ALD who developed bleeding problems on this diet, and in whom tests suggested a defect in vascular wall function or in platelet-endothelial interaction.

\section{Case report}

A 37-year-old man with adrenoleukodystrophy was placed on dietary therapy that restricted total fat intake to $15 \%$ of calories and added glycerol trioleate as $20 \%$ of total calories. ${ }^{5} \mathrm{~A}$ low-dose fish oil supplement was also started to avoid deficiencies ( $1 \mathrm{~g} /$ day; approximately $30 \%$ omega- 3 fatty acids). Other medications included cortisone, fludrocortisone, ranitidine, and baclofen. Platelet count was $242 \times 10^{9} / 1$ at initiation of dietary therapy and did not change during the following eight months. Treatment was then switched to Lorenzo's oil $(80 \%$ glycerol trioleate and $20 \%$ glycerol trierucate) when platelets were $230 \times 10^{9} / 1$ (table). Four weeks later the platelet count had decreased to $133 \times 10^{9} / 1$. The patient was started on ketorolac trimethamine (10 $\mathrm{mg}$ bid) for neuropathic pain and two days later he developed a spontaneous petechial rash on the right forearm. Platelet count was $108 \times 10^{9} / 1$. Ketorolac was discontinued, Lorenzo's oil was switched back to glycerol trioleate, and platelet count returned to pretreatment values within two weeks.

Lorenzo's oil was reintroduced when platelet count was $254 \times 10^{9} / 1$ and conventional platelet aggregation studies performed as a baseline were normal. At $5 \%$ of calories platelet count decreased to a low of $161 \times 10^{9} / 1$. Lorenzo's oil was then increased incrementally every four weeks. At $10 \%$ of calories platelets declined to $136 \times 10^{9} / 1$, while platelet function studies remained normal. Platelet function was also

\begin{tabular}{|l|}
\hline Adrenoleukodystrophy \\
\hline - inheritance: X-linked co-dominant \\
- cause of tissue damage: accumulation of \\
VLCFAs \\
- genetic defect: abnormal peroxisomal \\
ATP-binding transporter \\
- phenotypes: \\
cerebral form: brain affected, male infants and \\
children \\
adrenomyeloneuropathy: spinal cord and \\
peripheral nerves affected, male adolescents \\
and adults \\
adrenal and testicular failure: occurs in either \\
type \\
mothers of affected males: multiple \\
sclerosis-like manifestations, endocrine \\
abnormalities uncommon \\
- diagnosis: serum LCFAs elevated \\
treatment: none proven
\end{tabular}

Box 1

\section{Lorenzo's oil}

- composition: LCFAs, $80^{\circ}$ o glycerol trioleate, $20 \%$ glycerol trierucate

- theory: competes with VLCFAs which do not accumulate, tissue damage avoided

- evidence of effectiveness: marked decline of serum VLCFAs; positive clinical response in some anecdotal cases 
Table Haematological parameters during administration of Lorenzo's oil

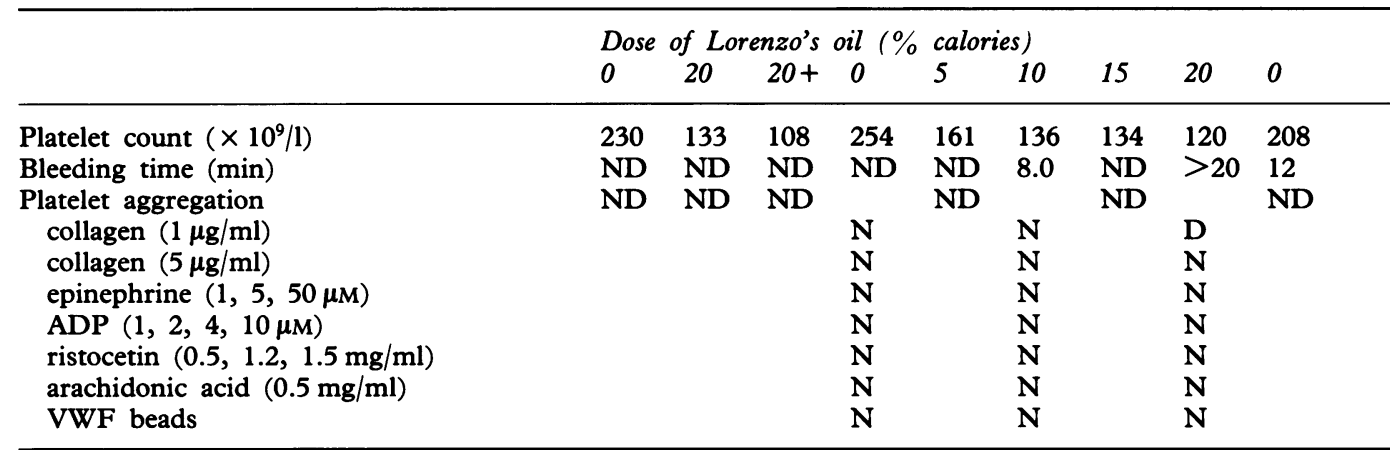

$t=$ values when ketorolac was added to therapy; $N D=$ not determined; $N=$ normal; $D=$ very slightly decreased; $\mathrm{ADP}=$ adenosine diphosphate; VWF = von Willebrand's factor

evaluated at this time by a recently described technique utilising von Willebrand factor immobilised onto polystyrene beads. ${ }^{6}$ In this method, platelet activation is assessed by measuring adenosine triphosphate release from platelet-dense granules induced by the beads. The patient's results were within the established normal range $(n=60)$. A bleeding time done at this point was at the upper range of normal at $8.0 \mathrm{~min}$. There were no bleeding episodes until Lorenzo's oil was increased to $20 \%$ of calories. Daily nose bleeds occurred with no local pathology. Haemorrhoids, which had previously bled only rarely, began to ooze blood at each bowel movement, with one large volume bleed. Bruising and petechiae occurred on various areas of the skin with either no or minimal trauma. Platelet count was minimally changed from values during the previous doses of Lorenzo's oil at $120 \times 10^{9} / 1$, and von Willebrand's factor bead-induced aggregation and conventional platelet aggregation studies remained normal except for a borderline response to low concentration collagen $(1 \mu \mathrm{g} / \mathrm{ml})$. The bleeding time, however, was elevated at greater than 20 minutes. Lorenzo's oil was discontinued, and in three weeks bleeding time decreased to $12 \mathrm{~min}$ and platelets increased to $208 \times 10^{9} / 1$. He had no further bleeding. Prothrombin time and partial thromboplastin time were never abnormal. At no time did he take aspirin-containing medications.

\section{Discussion}

The first bleeding episode in our patient was probably the result of platelet dysfunction arising from the addition of ketorolac to

\footnotetext{
1 Moser HW, Bergin A, Naidu S, Ladenson PW. Adrenoleukodystrophy. Endocrinol Metab Clin North Am 1991; 20: 297-318.

2 Moser AB, Borel J, Odone A, et al. A new dietary therapy for adrenoleukodystrophy: biochemical and preliminary clinical adrenoleukodystrophy: biochemical and preliminary cli

3 Zinkham WH, Kickler T, Borel J, Moser HW. Lorenzo's oil and thrombocytopenia in patients with adrenoleukodystrophy. N Engl f Med 1993; 328: 1124-5.

4 Chai BC, Siminoski K. Thrombocytopenia associated with use of Lorenzo's oil. Am $\mathcal{F}$ Hematol 1993; 44: 290-1.

5 Van Duyn MA, Moser AE, Brown FR, Sacktor N, Liu A,

Moser HW. The design of a diet restricted in saturated very long-chain fatty acids: therapeutic application in adrenoleukodystrophy. Am $\mathcal{f}$ Clin Nutr 1984; 40: 277-84.

6 Stewart MW, Etches WS, Gordon PA, Mant MJ, Shaw ARE. Evaluation of platelet function using immobilized VWF under stirring conditions. Thromb Hemost 1993; 69: 1143.
}

therapy, since the petechiae occurred two days after the drug was begun, and stopped when it was discontinued. When Lorenzo's oil was subsequently reintroduced, significant clinical bleeding began again at a dose of $20 \%$ of calories. Platelet counts had declined but were minimally below the lower normal range, a level at which bleeding does not usually occur. ${ }^{7.8}$ Platelet aggregation studies were normal, except for a borderline response to low concentration collagen, which can be seen in individuals with no history of clinical bleeding as well as in people on fish-oil enriched diets. $^{7-10}$ Moreover, von Willebrand factor bead-induced platelet activation fell within normal limits. Previous studies have suggested that the von Willebrand factor bead methodology is more sensitive for subtle abnormalities of platelet activation than are conventional aggregation studies using soluble agonists. ${ }^{6}$ Bleeding time, however, had increased from $8.0 \mathrm{~min}$ on $10 \%$ Lorenzo's oil to more than $20 \mathrm{~min}$ at a $20 \%$ dose. This constellation of changes, which has not been reported previously for LCFA-supplemented diets (either for Lorenzo's oil ${ }^{3,4}$ or for fish oils ${ }^{8-10}$ ) suggests a defect in endothelial function or platelet-endothelial interaction.

Fish oils have been shown to alter production of a variety of endothelial and platelet factors that are involved in coagulation. These changes result in a mild prolongation of bleeding time that is rarely greater than 3 to $4 \mathrm{~min}$, and which does not produce clinical bleeding. ${ }^{7-10}$ Neither Lorenzo's oil nor erucic acid have been investigated for their effects on these endothelial and platelet parameters, but similar mechanisms are likely.

7 Goodnight SH, Harris WS, Connor WE. The effects of dietary $\infty 3$ fatty acids on platelet composition and function in man: a prospective, controlled study. Blood 1981; 58: $880-5$.

8 Goodnight SH. Effects of dietary fish oil and omega-3 fatty acids on platelets and blood vessels. Semin Thromb Hemost 1988; 14: 285-9.

9 Knapp HR, Reilly IAG, Alessandrini P, FitzGerald GA. In vivo indexes of platelet and vascular function during fish-oil administration in patients with atherosclerosis. $N$ Engl f Med 1986; 314: 937-42.

10 Goodnight SH. Mechanism of the antithrombotic effects of fish oil. Bailliere's Clin Haematol 1990; 3: 601-23. 